\title{
CONTAMINACIÓN SONORA Y PERCEPCION DEL APRENDIZAJE
}

\author{
Tula SANCHEZ GARCIA \\ Universidad Nacional Mayor de San Marcos \\ tula.sanchez1@unmsm.edu.pe \\ Lozano SÁNCHEZ CORTEZ \\ Universidad Nacional Mayor de San Marcos \\ Isanchezc@unmsm.edu.pe \\ Ofelia SANTOS JIMÉNEZ \\ Universidad Nacional Mayor de San Marcos \\ Ofelia.santos@unmsm.edu.pe
}

\section{RESUMEN}

La contaminación sonora tiene implicancias en los aprendizajes de los estudiantes y causa efecto muy nocivo en ambientes ruidosos sobre todo en el rendimiento académico; es que el ruido tiene la capacidad de atraer involuntariamente nuestra atención y, por lo tanto, es disruptivo en relación con las tareas que podemos estar realizando en un determinado momento. Entre los efectos negativos destacan, además de déficit de atención, aumento de la tasa de errores, imprecisión y falta de calidad en las respuestas emitidas, estados generales de ansiedad y sensación global de cansancio, que les causa inquietudes y altera la comunicación, tal factor son los ruidos de impacto tales como claxon, escapes de transportes, propaganda callejera. La productividad se da en función inversa al ruido que haya alrededor, es decir, a mayor ruido menor será el rendimiento de una persona. Dentro del oído, existen unas células sensoriales auditivas no mayores a 18 mil en cada oído, y pierden su capacidad de renovación debido a sonidos demasiados fuertes, y es una lástima ya que las otras células sensoriales como las de la lengua, nariz y el ojo, que constantemente se están renovando. Como consecuencia docentes y estudiantes elevan el tono de la voz, repiten el mensaje que se propone dar, terminado con disfonías o ronqueras frecuentes.

\section{PALABRAS CLAVES}

Contaminación sonora, percepción del aprendizaje

\section{NOISE POLLUTION AND PERCEPTION OF LEARNING}

\section{ABSTRACT}

Sound pollution has implications for student learning and causes a very harmful effect in noisy environments, especially academic performance; is that noise has the capacity to unintentionally attract our attention and, therefore, it is disruptive in relation to the tasks that we may be performing at a given moment. Among the 
negative effects, in addition to attention deficit, increase in the error rate, imprecision and lack of quality in the responses issued, general states of anxiety and overall feeling of tiredness, which cause them concerns and alter communication, such a factor are impact noises such as horn, transport leaks, street propaganda. Productivity is given in inverse function to the noise that is around, that is, the higher the noise, the lower the performance of a person. Inside the ear, there are auditory sensory cells no larger than 18 thousand in each ear, and they lose their capacity for renewal due to too loud sounds, and it is a shame since the other sensory cells such as those of the tongue, nose and eye, which are constantly being renewed. As a consequence, teachers and students raise the tone of the voice, repeat the message they intend to give, ending with frequent hoarseness or hoarseness.

\section{KEY WORDS}

Sound pollution, learning perception

Recibido: $10 / 04 / 2020$

Aprobado: 29/06/2020

\section{OBJETIVO DE ESTUDIO}

stablecer la relación que existe entre la contaminación sonora y el aprendizaje según la percepción de los estudiantes del área de Ciencias Básicas de la Escuela de Estudios Generales en el semestre 2019-Il de la UNMSM

\section{Diseño}

La investigación corresponde al tipo aplicada, no experimental, nivel descriptivo, con diseño correlacional, en razón que describe la relación entre ellas.

El diagrama de investigación que asume es el siguiente:

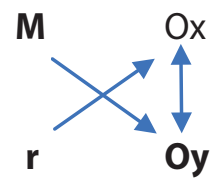

Donde:

M es la muestra de investigación.

Ox es la observación de la variable: Contaminación sonora.

Oy es la observación de la variable: Percepción del estudiante.

$r$ es la implicancia entre variables. 


\section{TÉCNICAS E INSTRUMENTOS DE RECOLECCIÓN DE DATOS}

Para la variable contaminación sonora se utilizó el método gráfico, que, por medio de toma de datos de diferentes ubicaciones, en diferentes tiempos te da un mapeo del ruido en una zona determinada previamente teniendo como programa computacional el "ArcGIS" permitiéndonos la visualización del ruido en zonas demarcadas por colores según la intensidad marcada por la toma de dato, y el instrumento fue el sonómetro, que es un equipo que registra los niveles de decídeles, haciendo un registro en determinadas horas.

Para la variable percepción del aprendizaje se utilizó la técnica de la encuesta, aplicando el cuestionario a los estudiantes de la muestra seleccionada.

\section{Validez de los instrumentos}

Se define validación de los instrumentos como la determinación de la capacidad de los instrumentos para medir cualidades para los cuales fueron construidos. Por cuanto este proceso se dio por juicio de expertos, para lo cual recurrimos a la opinión de tres doctores de la Universidad Nacional de San Marcos quienes determinaron la pertinencia muestral de los instrumentos. A ellos se les entrego la matriz de consistencia, los instrumentos y la ficha de validación donde se determinaron los indicadores respetivos.

Sobre la base del procedimiento de validación descrita, los expertos consideraron la existencia de una estrecha relación entre los criterios y objetivos del estudio en los ítems constitutivos de los dos instrumentos de recopilación de la información.

\section{Confiabilidad de los instrumentos}

Para la validación de los instrumentos se seleccionó una muestra piloto de 14 estudiantes y se les aplico el cuestionario con la finalidad de medir la comprensión y entendimiento de los ítems, ver la relación que guardan entre sí y comprobar si responde a los objetivos planteados, también se estimar los tiempos de aplicación del cuestionario y se detectó ciertos inconvenientes que se pueden presentar al momento de su aplicación.

Una vez recopilada y procesada la información, se hizo la validación mediante la confiabilidad del Alfa de Cronbach, estadístico desarrollado por J.L. Cronbach, que sirve para ver qué tan confiable es el instrumento, sus valores varían entre 0 y 1 , los valores que se aproximan a 0 indica baja confiabilidad y los valores que se aproximan a 1 indican alta confiabilidad. Esta estadística también sirve para conocer como mejoraría (o empeoraría) la confiabilidad del instrumento si se excluyera un determinado ítem con baja correlación. El Alfa de Cronbach se determina por la siguiente fórmula:

$$
\alpha=\kappa \frac{}{\kappa-1}\left(1-\Sigma S_{i}^{2} / S_{t}^{2}\right)
$$


Donde:

$$
\begin{array}{ll}
\kappa & =\text { Número de ítems } \\
S_{i}{ }^{2} & =\text { Varianza de las calificaciones en los ítems } \\
S_{t}{ }^{2} & =\text { calificaciones totales de la encuesta }
\end{array}
$$

Tabla 1. Criterios de interpretación del coeficiente del Alfa de Cronbach

\begin{tabular}{|l|c|c|c|}
\hline Muy baja & 0.01 & $\mathrm{a}$ & 0.20 \\
\hline Baja & 0.21 & $\mathrm{a}$ & 0.40 \\
\hline Moderada & 0.41 & $\mathrm{a}$ & 0.60 \\
\hline Alta & 0.61 & $\mathrm{a}$ & 0.80 \\
\hline Muy alta & 0.81 & $\mathrm{a}$ & 1 \\
\hline
\end{tabular}

Fuente: Ruiz Bolívar (2002

\section{ANÁLISIS E INTERPRETACIÓN DE RESULTADOS}

Para demostrar los objetivos y dar respuesta a las hipótesis se recogieron muestras del ruido ambiental en puntos estratégicos de la UNMSM y se encuesto a los estudiantes para conocer acerca de la percepción de la contaminación sonora y de su aprendizaje a causa de factores del ruido ambiental.

Los resultados se presentan mediante un análisis descriptivo variable por variable, para conocer los niveles de contaminación sonora y los niveles de percepción del aprendizaje, y mediante un análisis estadístico de la correlación de Spearman para dar respuesta a las hipótesis.

\section{HIPÓTESIS DE ESTUDIO}

La hipótesis se comprobó mediante el estadístico de la correlación Rho de Spearman, que es una prueba no paramétrica que sirve para ver la relación que existe entre dos variables cuando sus datos se encuentran en escala ordinal o no existe distribución normal.

Para el estudio las variables están medidas en escala ordinal. Contaminación sonora: 1 = baja, 2 = medio, 3 = alta y $4=$ muy alta; Percepción del aprendizaje: 1 = Deficiente, 2 = Bajo, 3 = Medio y $4=$ alto, lo cual cumple los supuestos para utilizar el estadístico Rho de Spearman

La contaminación sonora tiene una relación negativa con el aprendizaje según la percepción de los estudiantes del área de ciencias básicas de la escuela de estudios generales en el semestre 2019-II de la UNMSM 
$H_{o}: \rho=0$ : La contaminación sonora no tiene una relación negativa con el aprendizaje según la percepción de los estudiantes del área de ciencias básicas de la escuela de estudios generales en el semestre 2019-II de la UNMSM.

$H_{1}: \rho \neq 0$ : La contaminación sonora tiene una relación negativa con el aprendizaje según la percepción de los estudiantes del área de ciencias básicas de la escuela de estudios generales en el semestre 2019-II de la UNMSM.

Nivel de significancia: $\alpha=0.05$ (si la sig. Es $\leq 0.05$ se rechaza $H_{0}$ )

Tabla 2. Correlación entre Contaminación sonora y el aprendizaje según la percepción de los estudiantes.

\begin{tabular}{|l|c|}
\hline & Valores \\
\hline Coeficiente de correlación Rho de Spearman & $-0,724$ \\
Sig. (bilateral) & 0,000 \\
N de casos & 80 \\
\hline
\end{tabular}

Fuente: Elaboraciòn propia

El coeficiente de correlación Rho de Spearman es de -0.724, lo que indica que si existe una correlación moderada e inversa entre la contaminación sonora y el aprendizaje según la percepción de los estudiantes y como la sig $=0.00$, esta correlación es significativa, por lo que se rechaza $\mathrm{H}_{0}$ y se acepta $\mathrm{H}_{1}$, lo que permite afirmar a un $95 \%$ de confianza que la contaminación sonora tiene una relación negativa moderada con el aprendizaje según la percepción de los estudiantes del área de ciencias básicas de la escuela de estudios generales en el semestre 2019-II de la UNMSM, es decir la contaminación sonora influye de forma negativa en el aprendizaje, ya que, altos niveles de ruido ambiental afectan en el desempeño y en las actitudes de los estudiantes, no permite la concentración adecuada, entender a cabalidad la explicación de sus docentes, afecta en los trabajos en equipo, les genera cambios de humor, interfiere y les provoca alteraciones para comunicarse con sus compañeros, les provoca estrés y alteraciones en su sistema nervios.

\section{DISCUSIÓN DE RESULTADOS}

De los resultados producto del trabajo de campo: Muestras tomadas para determinar la contaminación sonora y las encuestas aplicadas a los estudiantes se ha determinado:

De las muestras recogidas del ruido ambiental en el frontis de la Facultad de Ciencias Matemáticas y en el Frontis de la Red Telemática se ha determinado que, si existe contaminación sonora, esto debido a que los niveles de ruido ambiental superan los decibeles permisibles para zonas de protección especial 
establecidos por los Estándares Nacionales de Calidad Ambiental. En el frontis de la Facultad de Ciencias Matemáticas de $8 \mathrm{~h}$ a $14 \mathrm{~h}$ el ruido ambiental es molesto, las puntuaciones promedio varían entre 53.4 y 59.6 decibeles y las puntuaciones máximas que varían entre 62.1 y 69.3 decibles, de las $14 \mathrm{~h}$ a $20 \mathrm{~h}$ el ruido ambiental aumenta llegando en algunos horarios a ser un ruido nocivo perjudicial para la salud, en algunos horarios alcanza hasta 82.8 decibeles. En el frontis de la Red Telemática el ruido ambiental también es molesto, pero es más bajo que en el frontis de la Facultad de Ciencias Matemáticas, aquí el ruido ambiental promedio de $8 \mathrm{~h}$ a $14 \mathrm{~h}$ se mantiene casi constante varía entre 51.1 y 53.4 decibeles luego a partir de las $14 \mathrm{~h}$ empieza aumentar llegando a las $20 \mathrm{~h}$ a 65.4 decibeles.

En la Facultad de Ciencias Matemáticas el 33\% de estudiantes perciben que la contaminación sonora es alta y el $30 \%$ perciben una contaminación sonora muy alta, mientras que en la Facultad de Ciencias Físicas el $43 \%$ de estudiantes perciben que la contaminación sonora es alta y solo el $13 \%$ perciben que la contaminación sonora es muy alta y en la Facultad de Ciencias Biológicas el 55\% de estudiantes perciben que la contaminación sonora es alta y el 10\% perciben que la contaminación sonora es muy alta.

Mediante la estadística Rho de Spearmana un 95\% de confianza, se ha determinado que existe una relación moderada y negativa entre la contaminación sonora y la percepción del aprendizaje de los estudiantes del área de Ciencias Básicas de la Escuela de Estudios Generales en el semestre 2019-II de la UNMSM, el coeficiente de correlación es de - 0.724 con una sig. 0.00 .

La contaminación sonora afecta de forma negativa en el aprendizaje, ya que se ha determinado que la contaminación sonora afectan en el desempeño y en las actitudes de los estudiantes, debido a que la mayoría de ellos manifiestan que altos niveles de ruido ambiental: no les permite una concentración adecuada, entender a cabalidad la explicación de sus docentes, les afecta al realizar sus trabajos en equipo, les genera cambios de humor, interfiere y les provoca alteraciones para comunicarse con sus compañeros, les provoca estrés y alteraciones en su sistema nervios.

Mediante la estadística Rho de Spearman a un 95\% de confianza, se ha determinado que existe moderada y negativa relación entre la contaminación sonora y el desempeño de los estudiantes del área de ciencias básicas de la escuela de estudios generales en el semestre 2019-II de la UNMSM, el coeficiente de correlación es de -0.671 con una sig. 0.00 .

Mediante la estadística Rho de Spearman a un 95\% de confianza, se ha determinado que existe relación moderada y negativa entre la contaminación sonora y las actitudes de los estudiantes del área de ciencias básicas de la escuela de estudios generales en el semestre 2019-II de la UNMSM, el coeficiente de correlación es de -0,592 con una sig. 0.00 . 


\section{CONCLUSIONES}

De los resultados producto del trabajo de campo: Muestras tomadas para determinar la contaminación sonora y las encuestas aplicadas a los estudiantes ,se ha determinado:

1. De las muestras recogidas del ruido ambiental en el frontis de la Facultad de Ciencias Matemáticas y en el Frontis de la Red Telemática se ha determinado que, si existe contaminación sonora, esto debido a que los niveles de ruido ambiental superan los decibeles permisibles para zonas de protección especial establecidos por los Estándares Nacionales de Calidad Ambiental. En el frontis de la Facultad de Ciencias Matemáticas de $8 \mathrm{~h}$ a $14 \mathrm{~h}$ el ruido ambiental es molesto, las puntuaciones promedio varían entre 53.4 y 59.6 decibeles y las puntuaciones máximas que varían entre 62.1 y 69.3 decibles, de las $14 \mathrm{~h}$ a $20 \mathrm{~h}$ el ruido ambiental aumenta llegando en algunos horarios a ser un ruido nocivo perjudicial para la salud, en algunos horarios alcanza hasta 82.8 decibeles. En el frontis de la Red Telemática el ruido ambiental también es molesto, pero es más bajo que $\mathrm{n}$ el frontis de la Facultad de Ciencias Matemáticas, aquí el ruido ambiental promedio de $8 \mathrm{~h}$ a $14 \mathrm{~h}$ se mantiene casi constante varía entre 51.1 y 53.4 decibeles luego a partir de las $14 \mathrm{~h}$ empieza aumentar llegando a las $20 \mathrm{~h}$ a 65.4 decibeles.

2. Respecto a la percepción de los estudiantes acerca del ruido ambiental en la Facultad de Ciencias Matemáticas, el 33\% de estudiantes perciben que la contaminación sonora es alta y el $30 \%$ perciben una contaminación sonora muy alta, mientras que en la Facultad de Ciencias Físicas el $43 \%$ de estudiantes perciben que la contaminación sonora es alta y solo el 13\% perciben que la contaminación sonora es muy alta y en la Facultad de Ciencias Biológicas el $55 \%$ de estudiantes perciben que la contaminación sonora es alta y el $10 \%$ perciben que la contaminación sonora es muy alta.

\section{REFERENCIAS BIBLIOGRÁFICAS}

Amable Álvarez I, Méndez Martínez J, Delgado Pérez L, Acebo Figueroa F, de Armas Mestre J, Rivero Llop ML. (2017). Contaminación ambiental por ruido. Rev Méd Electrón [Internet]. May-Jun [citado: fecha de acceso];39(3). Disponible en: http://www.revmedicaelectronica.sld.cu/index.php/rme/ article/view/2305/3446

Bernal, C. (2006). Metodología de la investigación. México: Pearson Educación de México.

Campos, V (2000). Procesos Cognoscitivos y Afectivos. Universidad Autónoma de Barcelona. Tesis de Grado para optar al Título de Magíster en Psicología Social.

Jimena Martínez Llorente Y Jens Peters (2015). Contaminación acústica y ruido. 
OEFA-Organismo de Evaluación y Fiscalización Ambiental (2016). La contaminación sonora en Lima y Callao.

OMS - Organización Mundial de la Salud (2013). Datos y estadísticas. Organización Mundial de la salud, Oficina Regional para Europa Accesible: http://www.euro. who.int/en/health-topics/environment-and-health/noise/data-andstatistics. [Último acceso: octubre 2015].

Ortiz A. (2009). Aprendizaje y Comportamiento basados en el funcionamiento del cerebro humano, ediciones litoral.

Ramírez L. (2012). Estudio comparativo de contaminación sonora entre los estándares permisibles y lo real en la ciudad de lquitos, tesis para optar el título de Ingeniero en Gestión ambiental.

RUIZ BOLIVAR, Carlos. Instrumentos de Investigación Educativa: procedimientos para su diseño y validación. 2 ed. Barquisemeto, Venezuela: Ediciones CIDEG, C.A. p. 102-107 [citado 2010-02-05]. Disponible en Internet: 226 p. 